$1.31 ; 95 \% \mathrm{Cl}, 1.05$ to 1.63$)$. Further adjustment by body-mass index (BMI) attenuated these associations to non-significance for both sexes (table 1). AA females had a higher odds of hyperuricemia than White females (age-adjusted OR, 2.17; $95 \% \mathrm{Cl}, 1.72$ to 2.73 ), but not males (age-adjusted OR, $1.08 ; 95 \% \mathrm{Cl}, 0.95$ to 1.22; $P$ for interaction $=0.002$ ) (table 1 ). Among females, this association attenuated after further adjustment for BMI, hypertension, CKD, type 2 diabetes mellitus, household income, and education, but remained significant (adjusted OR, 1.52; $95 \% \mathrm{Cl}, 1.19$ to 1.95$)$.

Abstract SAT0703 - Table 1. ORs of Gout and Hyperuricemia According Race/Ethnicity, NHANES 2007-16

\begin{tabular}{|c|c|c|c|c|}
\hline & \multicolumn{2}{|c|}{ Gout } & \multicolumn{2}{|c|}{ Hyperuricemia } \\
\hline & $\begin{array}{l}\text { Age-adjusted } \\
\text { OR }(95 \% \mathrm{Cl})\end{array}$ & $\begin{array}{l}\text { Age and } \mathrm{BMI}- \\
\text { adjusted OR } \\
(95 \% \mathrm{Cl})\end{array}$ & $\begin{array}{l}\text { Age-adjusted } \\
\text { OR }(95 \% \mathrm{Cl})\end{array}$ & $\begin{array}{l}\text { Age and BMl- } \\
\text { adjusted OR } \\
(95 \% \mathrm{Cl})\end{array}$ \\
\hline \multicolumn{5}{|l|}{ Female } \\
\hline White & 1.00 (Referent) & 1.00 (Referent) & 1.00 (Referent) & 1.00 (Referent) \\
\hline $\begin{array}{l}\text { African } \\
\text { American }\end{array}$ & $\begin{array}{c}1.65(1.14- \\
2.38)\end{array}$ & $1.33(0.92-1.92)$ & $\begin{array}{c}2.17(1.72- \\
2.73)\end{array}$ & $1.62(1.27-2.05)$ \\
\hline \multicolumn{5}{|l|}{ Male } \\
\hline White & 1.00 (Referent) & 1.00 (Referent) & 1.00 (Referent) & 1.00 (Referent) \\
\hline $\begin{array}{l}\text { African } \\
\text { American }\end{array}$ & $\begin{array}{c}1.31(1.05- \\
1.63)\end{array}$ & $1.24(1.00-1.53)$ & $\begin{array}{c}1.08(0.95- \\
1.22)\end{array}$ & $1.02(0.89-1.17)$ \\
\hline
\end{tabular}

Conclusions: These nationally-representative data indicate that AAs have a larger disease burden of gout and hyperuricemia than Whites, particularly among women. This burden appears to be at least partly due to a higher prevalence of risk factors for hyperuricemia in AAs. ${ }^{1}$

REFERENCE:

[1] Flegal KM, et al. JAMA 2016 Jun 7;315(21):2284-2291.

Disclosure of Interest: M. Chen-Xu: None declared, C. Yokose: None declared, H. Choi Grant/research support from: Ironwood and Ardea/AstraZeneca, Consultant for: Horizon and Selecta

DOI: 10.1136/annrheumdis-2018-eular.6059

\section{SAT0704 COLLECTION OF ANTI-RHEUMATIC MEDICATION DATA FROM BOTH PATIENTS AND RHEUMATOLOGISTS SHOWS STRONG AGREEMENT IN A REAL WORLD CLINICAL COHORT: RESULTS FROM THE ONTARIO BEST PRACTICES RESEARCH INITIATIVE (OBRI)}

M. Movahedi ${ }^{1}$, A. Cesta ${ }^{1}$, X. Li ${ }^{1}$, C. Bombardier ${ }^{1,2,3}$ on behalf of Other OBRI Investigators. ${ }^{1}$ Ontario Best Practices Research Initiative, Toronro General Research Insititute, University Health Network, ${ }^{2}$ Department of Medicine (DOM) and Institute of Health Policy, Management, and Evaluation (IHPME), University of Toronto; ${ }^{3}$ Division of Rheumatology, Mount Sinai Hospital, Toronto, Canada

Background: Collection of Anti-Rheumatic Medication (ARM) information from both patients and rheumatologists is considered a strength for Rheumatoid Arthritis (RA) registries and cohorts. However, it is important to assess the agreement between these two data sources.

Objectives: We aimed to examine the agreement of ARM use, their administration routes, and start and stop dates between self-reports and rheumatologist reports in the Ontario Best Practices Research Initiative (OBRI).

Methods: Adult Patients enrolled in the OBRI who consented to both patient interviews and rheumatologist evaluations were included. Patients in the OBRI are interviewed every six months, while rheumatologist assessments are conducted as per routine care. For this analysis, we included patients who enrolled in OBRI on or after Sep 1 st 2010 and compared ARM use reports where rheumatologist visits and interviews occurred within 60 days of each other. ARM included conventional synthetic Disease-Modifying Antirheumatic Drugs (csDMARDs) and biologic DMARDs (bDMARDs). Cohens' Kappa statistics of agreement between the two data sources were calculated. Kappa values $0.61-0.80$ were considered to represent good and $0.81-1.00$ as very good agreement. To examine factors associated with agreement, a multivariate backward stepwise logistic regression was used to model the odds of agreement for ARM use. The agreement and absolute time gap (days) for starts and stops dates between self-reports and rheumatologist reports were also assessed and presented by median and interquartile range (IQR) in a subset analysis.

Results: 2154 patients ( $78.7 \%$ female) were included with a mean (SD) age at OBRI enrolment of 57.8 (12.6) year. Mean (SD) disease parameters were: disease duration: 8.4 years (9.9); DAS28: 4.2 (1.6); physician global: 4.0 (2.5); and health assessment questionnaire (HAQ) disability Index: 1.1 (0.8). For csDMARDs use, the prevalence was $74.2 \%$ based on self-reports and $76.6 \%$ based on rheumatologist reports. The prevalence of bDMARDs use was approximately $20.0 \%$ based on both reports.

Overall agreement for ARM use between self-reports and rheumatologist reports was good. In the regression model, increased HAQ-pain index (OR: 0.66 95\% Cl: 0.60-0.73) and physician global (OR: 0.95; 95\% Cl: 0.92-0.98) were sig nificantly associated with the lower agreement. By contrast, post-secondary edu cation (OR: 1.20; 95\% Cl: 1.02-1.40), and seeing an academic rheumatologist (OR: $1.47 ; 95 \% \mathrm{Cl}: 1.25-1.73$ ) were significantly associated with the higher agreement between two data sources.

There was a good and very good agreement for reported administration route of bDMARDs and csDMARDs, respectively. The median absolute time gap (IQR) of start dates and stop dates for ARM use reported by two data sources was 7 days 1-27 and 19 days, 5-48 respectively.

Conclusions: The results of this analysis suggest that ARM reports from the two data sources have strong agreement in the OBRI. This agreement is even better for patients who have post-secondary education and are being treated by an academic rheumatologist.

Disclosure of Interest: M. Movahedi Employee of: OBRI, A. Cesta Employee of: OBRI, X. Li Employee of: OBRI, C. Bombardier Grant/research support from: OBRI was funded by peer reviewed grants from $\mathrm{ClHR}$ (Canadian Institute for Health Research), Ontario Ministry of Health and Long-Term Care (MOHLTC), Canadian Arthritis Network (CAN) and unrestricted grants from: Abbvie, Amgen, Bristol Myers Squibb, Celgene, Hospira, Janssen, Pfizer, Roche, and UCB, Consultant for: Dr. Bombardier holds a Canada Research Chair in Knowledge Transfer for Musculoskeletal Care and a Pfizer Research Chair in Rheumatology DOI: 10.1136/annrheumdis-2018-eular.2136

\section{SAT0705 ASSOCIATION BETWEEN FRACTURE SITES IN PATIENTS WITH A HISTORY OF PARENTAL FRACTURE}

M. Dey, M. Bukhari. Rheumatology, University Hospitals of Morecambe Bay NHS Foundation Trust, Lancaster, UK

Background: Fragility fractures (FF) are fractures due to low energy force. Factors predisposing to FF in the general population include reduced bone minera density (BMD), and family history of osteoporosis. FF most commonly occur in the vertebrae, proximal femur, and distal radius. Studies have demonstrated increased risk of $F F$ in patients with decreased $B M D$ and parental history of FF, particularly hip fracture. ${ }^{1,2}$ Few data exist on the association between sites of fracture in patients with a history of parental fracture, especially whether they co-exist at several sites and if particular factors are associated with discrete sites.

Objectives: We aimed to find the correlation between sites of FF in patients with a history of parental fracture, and identify and examine the clinical association with any clusters of fractures.

Methods: 2094 patients with a history of parental FF and personal history of at least one FF, presenting for BMD estimation from their primary or secondary care practitioner, from 2006-2016, were included. Parameters recorded: height, weight, age at scan, average fat mass, site of fracture(s), smoking, alcohol, corticosteroid use, aromatase inhibitor use, Depo-Provera use, hormone replacement therapy (HRT), rheumatoid arthritis (RA), polymyalgia rheumatica (PMR), breast or prostate cancer, and coeliac disease.

Factor analyses with polychoric correlation matrices were applied to determine association between fracture sites. Any associations with Eigenvalues of more than one were then examined using a logistic model to analyse the effect of the above risk factors.

Results: Fracture sites with Eigenvalue of more than one (tibia/fibula, spine, ribs, pelvis) were compared to sites with least co-variability (humerus, forearm, femur) The two cohorts were significantly different in age; therefore, an age-adjusted model is reported below (table 1). Smoking, HRT, and increased age significantly impacted clustering of fractures in the tibia/fibula, spine, ribs, and pelvis, compared with clustering at the humerus, forearm, and femur.

Abstract SAT0705 - Table 1. Age-adjusted predictors of fracture for tibia/fibula/spine/ribs/ pelvis vs. humerus/forearm/femur ( ${ }^{*}$ denotes significance)

\begin{tabular}{lc}
\hline Variable/Fracture cluster & OR (95\% Cl) \\
\hline Corticosteroid & $0.878[0.748-1.031]$ \\
Smoking & $0.879[0.779,0.992]$ \\
Alcohol & $0.954[0.808-1.127]$ \\
Rheumatoid arthritis & $1.393[0.928-2.092]$ \\
Polymyalgia rheumatica & $0.907[0.465-1.769]$ \\
HRT & $0.635[0.420,0.961]^{*}$ \\
Aromatase inhibitors & $0.950[0.772,1.170]$ \\
Breast/prostate cancer & $1.489[0.610-3.636]$ \\
Gender & $0.804[0.589-1.096]$ \\
Age at scan (years) & $1.011[1.003,1.019]^{*}$ \\
Height $(\mathrm{cm})$ & $0.989[0.978-1.000]$ \\
Weight $(\mathrm{kg})$ & $0.995[0.989-1.000]$ \\
\hline
\end{tabular}


Conclusions: In this cohort of patients, there was overlap between all fracture sites, with significant clustering seen in fractures of the tibia/fibula, spine, ribs, and pelvis. After adjusting for age, predictors of fracture in this cluster were smoking, HRT and increased age. This indicates that risk factors for FF are different at different sites, and affects the association of fracture between sites. Further work validating this finding is currently underway.

\section{REFERENCES:}

[1] A family history of fracture and fracture risk: a meta-analysis. Bone [Internet]. Elsevier 2004 Nov 1 [cited 2017 Oct 27];35(5):1029-37.

[2] Strong familial association of bone mineral density between parents and offspring: KNHANES 2008-2011. Osteoporos Int 2017.

Disclosure of Interest: None declared

DOI: 10.1136/annrheumdis-2018-eular.2447

\section{SAT0706 INCIDENCE OF PSORIATIC ARTHRITIS IN GERMANY: ANALYSIS OF CLAIMS DATA FROM 65 MILLION PEOPLE FROM 2009 TO 2012}

P. Sewerin, I. Haase, M. Schneider, B. Ostendorf, R. Brinks. Department for Rheumatology, University Hospital Duesseldorf, Düsseldorf, Germany

Background: Epidemiological studies are important for understanding the etiology and burden of psoriatic arthritis (PsA). Currently, there are no data available about the incidence of PsA in Germany.

Objectives: This study aims to estimate the age-standardised incidence of diagnosed PsA for German men and women during 2009 to 2012.

Methods: Estimation of the incidence of a chronic disease from prevalence data is possible if information about the general mortality and excess mortality of diseased compared to non-diseased people are available in terms of the hazard ratio (HR). ${ }^{1}$ Prevalence was extracted from the complete diagnosis data (in- and outpatient) from about $80 \%$ of the overall German population during 2009 to 2012 . Diagnoses are based on claims data from all insurances of the German statutory health insurance (SHI) system. After determining the age-standardised sex-specific prevalence of PsA for each of the four years, the age-standardised incidence for men and women has been estimated. General mortality was obtained from the Federal Statistical Office of Germany. Since the HR is unknown in Germany, we use different scenarios motivated from a systematic review ${ }^{2}$ in the range from 1.3 to 1.6 .

Results: For each of the years from 2009 to 2012, a total of $127,138,146$ and 156 thousand patients with diagnosed PsA were identified in about 65 million people from the SHI, respectively. The age-standardised prevalence increases from 1.8 to 2.1 per mil in men, and from 2.1 to 2.5 per mil in women. The estimated agestandardised incidence over the study period is shown in the figure 1. Over the study period, the incidence rate of PsA decreases for both sexes and the rate of men is lower than the rate of women. The average incidence rates are 11.5 and 14.5 per 100 '000 person-years for men and women, respectively. This means that about 4700 men and 5900 women contract PsA each year. The impact of the different scenarios in HR is small.

Conclusions: These data from about 65 million people insured in the German $\mathrm{SHI}$ for the first time allow an estimation of the incidence of PsA in Germany. A selection bias is likely to be present, because the roughly $20 \%$ of the overall German population who could not included in the analysis (mainly privately insured people) are known to have other health risks. However, the results refer to the vast majority of the German population. The analysis cannot be adjusted for potential confounders other than age and sex (e.g., socio-economic position or presence of co-morbidities)

\section{REFERENCES:}

[1] Brinks R, Landwehr S, DOI: 10.1093/imammb/dqu024

[2] Gladman DD. Mortality in psoriatic arthritis, Clin Experim Rheuma 26(5): S62.

Disclosure of Interest: None declared

DOI: 10.1136/annrheumdis-2018-eular.2717

\section{SAT0707 \\ TRENDS IN THE INCIDENCE OF RHEUMATOID ARTHRITIS IN DENMARK FROM 1995-2016: A NATIONWIDE REGISTER-BASED STUDY}

R.L. Cordtz ${ }^{1}$, P. Højgaard ${ }^{1,2}$, K. Zobbe ${ }^{1,2}$, L. Dreyer ${ }^{1,2,3} .{ }^{1}$ Center for Rheumatology and Spine Diseases, Rigshospitalet- Gentofte, Copenhagen; ${ }^{2}$ The Parker Institute, Bispebjerg and Frederiksberg Hospital, Frederiksberg; ${ }^{3}$ Department of Rheumatology, Aalborg University Hospital, Aalborg, Denmark

Background: Previous studies have shown conflicting results regarding temporal trends in the incidence of rheumatoid arthritis (RA)

Objectives: To investigate annual incidence rates of RA in Denmark from 19952016 using nationwide health care registers, and to explore how these are affected by different case definitions of RA

Methods: The Danish National Patient Register captures all in- and outpatient (since 1977 and 1994, respectively) contacts at private and public hospitals. Excluding prevalent cases (ICD-8: 712), and using two different case-definitions, we identified all incident RA patients (ICD-10 codes M05.1;3;8;9 and M06.0;8;9) aged $\geq 18$ in each year from 1995-2016, and calculated annual age-standardised incidence rates (per 100000 person years). We used the NORDCAN population for direct standardisation, and the number of adults alive in Denmark at the beginning of each year as the denominator. We furthermore calculated the age- and sex specific incidence rates during the period. Strict case definition: incident patients were required to have at least two in- or outpatient visits at a rheumatology- or general internal medicine clinic/department within 90 days with RA listed as the main diagnosis. Liberal case definition: patients with at least two in- or outpatient contacts listing RA as a main- or contributory diagnosis within 1 year. ${ }^{1}$

Results: We identified 26090 and 43080 patients using the Strict and Liberal case definitions, respectively. Patient characteristics according to choice of definition are presented in the Table. The overall incidence rates were $23.7 / 100,000$ person years (23.4-23.9) using the Strict and 39.7/100,000 person years (39.440.1) using the Liberal definition. As seen from the figure 1, a slightly increasing trend in RA was observed from 1995 to 2016 independent of choice of case definition, and this increase was more pronounced in 2010.

The age-specific incidence rates were highest in women (Strict: 75/100,000 person years; Liberal: $134 / 100,000$ person years) as well as men (Strict: $47 / 100,000$ person years; Liberal: 81/100,000 person years) aged 70-79 years.

Table. Demographics and characteristics of rheumatoid arthritis patients diagnosed at hospitals in Denmark 1995-2016 according to case definition.

\begin{tabular}{lcc}
\hline & Strict case definition & Liberal case definition \\
\hline $\mathrm{N}$ & 26090 & 43820 \\
Mean (s.d) age at diagnosis & $58.1(15.2)$ & $59.3(15.5)$ \\
Females, n (\%) & $18238(70)$ & $30749(70)$ \\
\% with COPD & 3.1 & 3.7 \\
\% with DM & 3.3 & 3.8 \\
\% with CVD & 7.2 & 8.4 \\
\hline
\end{tabular}

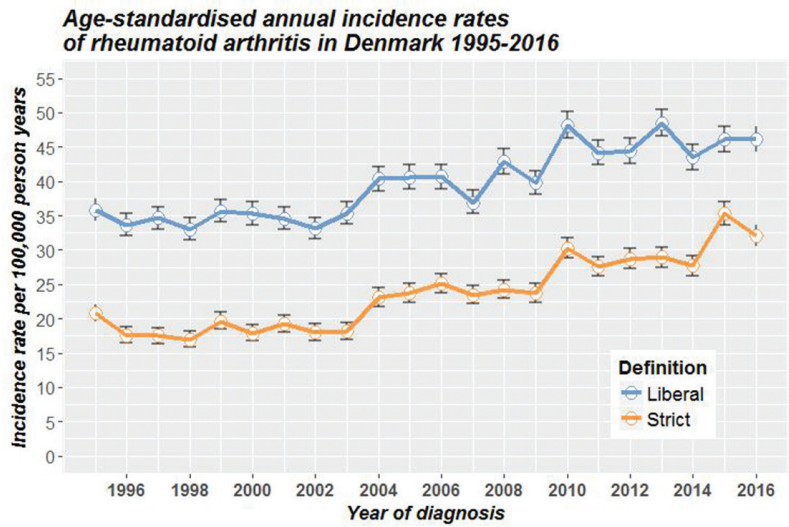

Conclusions: We found a slightly increasing trend in the incidence rate of RA from 1995-2016. Further, we found that using the Liberal case definition, the overall and age- and sex specific incidence rates were remarkably similar to those reported from Sweden ${ }^{1}$, whereas the Strict definition resulted in lower incidence rates than previously reported from other countries. 